\title{
A Compact Integrated 40Gb/s Packet Demultiplexer and Label Extractor on Silicon-on-Insulator for an Optical Packet Switch
}

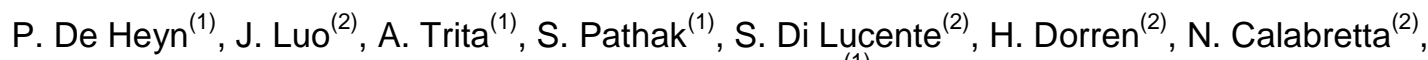 \\ D. Van Thourhout ${ }^{(1)}$ \\ (1) Photonics Research Group, Department of Information Technology, Ghent University - imec, Ghent \\ B-9000, Belgium, Peter.DeHeyn@intec.ugent.be \\ (2) Eindhoven University of Technology, Department of Electrical Engineering, 5600MB Eindhoven,
} The Netherlands

Abstract We demonstrate a compact 40Gb/s 32-channel packet demultiplexer and in-band label extractor based on photonic integrated AWG followed by a narrow-band microring resonator at each $A W G$ output. Error free operation with $\leq 0.5 d B$ penalty was measured.

\section{Introduction}

Several research projects $^{1}$ investigated large port-count and low latency optical packet switches (OPS) in order to flatten the data center network (DCN) and thus to eliminate the communication bottleneck of current DCNs tree topology. However, all the architectures presented employ a centralized controller that leads to port-count dependent reconfiguration time of the switch. Scaling these architectures to thousands of ports will result in high switch configuration time and thus high latency. The numerical and experimental demonstration of a novel modular WDM optical packet switch architecture was demonstrated ${ }^{2,3}$. Each module forwards the M WDM packets to the M output ports based on the information carried by the optical label. The optical label (one for each WDM payload) is transmitted in-band with the optical payload to maximize the optical spectral efficiency and to allow an asynchronous and easier extraction of the label. Each module includes an arrayed waveguide grating (AWG) WDM packet demultiplexer, narrow band fiber Bragg grating filters as optical label extractors, $1 \times N$ optical switches, and a switch controller (see Fig. 1).

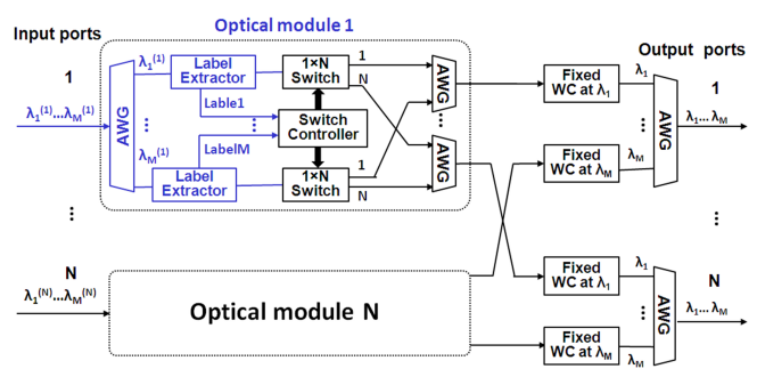

Fig. 1: Architecture of the modular WDM OPS with the demonstrated functionality in this work in blue..

Although the modular OPS architecture has been demonstrated by using off-the shelf discrete components, photonic integration of such complex systems is necessary to decrease drastically the size, unit cost and power consumption.

In this paper we bring the realization of an integrated full-functional OPS one step further by demonstrating a silicon photonic integrated device that includes a $32 \times 200 \mathrm{GHz}$ AWG $40 \mathrm{~Gb} / \mathrm{s}$ packet demultiplexer and a narrow band microring resonator (MRR) for label extraction at each of the AWG output waveguides. The chip is fabricated in the high-contrast material system silicon-on-insulator (SOI) to realize ultra compact circuits with small bending radii $(<5 \mu \mathrm{m})$.

Experimental results confirmed a channel crosstalk lower than $18 \mathrm{~dB}$ and error-free operation less than $0.5 \mathrm{~dB}$ penalty after the $\mathrm{AWG}$ and the label extractor filtering. Also the label, modulated at $1.3 \mathrm{~Gb} / \mathrm{s}$ has been error-free detected after the label extracted with a power penalty of $0.5 \mathrm{~dB}$.

\section{Fabrication, design and characterization of the photonic device}

Our design is fabricated on a SOl platform exhibiting a $220 \mathrm{~nm}$ thick silicon device layer on a $2 \mu \mathrm{m}$ thick buried oxide layer. The label extracting MRR must be very narrow band in order to not distort the payload and to minimize the crosstalk of the payload on the label. Using rib waveguides, one can reduce the influence of the sidewall roughness and therefore minimize backscattering which ultimately limits the $Q$ factor of a MRR on SOI using strip waveguides ${ }^{4}$. To realize the rib, a shallow etch step of $70 \mathrm{~nm}$ is used. This etch step is also used to realize input and output fiber grating couplers as well as the star-coupler of the $\mathrm{AWG}^{5}$. An AWG consist of two free propagation regions (which are known as star-couplers) and an array of waveguides with successive increment of length. We used shallowly etched waveguide apertures as inputs and outputs of the star-couplers to reduce the 
reflections. To design the $32 \times 200 \mathrm{GHz}$ AWG we used 90 waveguides in the array.

The radius of the MRR is $35 \mu \mathrm{m}$ which corresponds with a free spectral range of $2.92 \mathrm{~nm}$, which is large enough to avoid any extra distortion of the incoming payload.

Both components are characterized first isolated from each other and then cascaded using a tunable laser. In Fig. 2a, the optical spectrum of the isolated AWG is plotted. The center channel insertion loss is small $(-1.2 \mathrm{~dB})$ and the $1 \mathrm{~dB}$ bandwidth $(\mathrm{BW})$ is wide $(0.56 \mathrm{~nm})$. With a roll-off factor (defined as $1 \mathrm{~dB} B W / 10 \mathrm{~dB}$ BW) of 0.33 , and a crosstalk below $-18 \mathrm{~dB}$, this filter exhibits a channel response good enough to successfully demultiplex $40 \mathrm{~Gb} / \mathrm{s}$ signals.
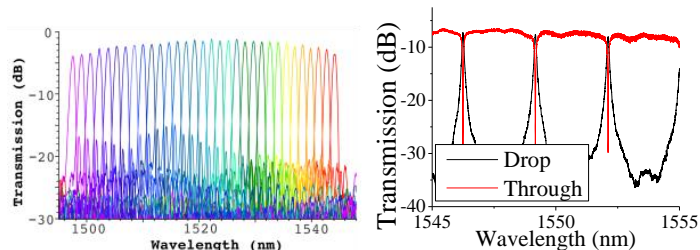

Fig. 2: Optical spectrum of a) the isolated $32 \times 200$ $\mathrm{GHz} A W G$ and $b$ ) the narrow-band MRR.

The optical spectrum of an isolated MRR is plotted in Fig. 2b. The extinction ratio (ER) of the through is large $(-22.5 \mathrm{~dB})$ and the IL for the drop small $(<0.5 \mathrm{~dB})$. The $3 \mathrm{~dB}$ bandwidth $(\mathrm{BW})$ is very narrow $(77 \mathrm{pm})$ to avoid distortion on the payload.

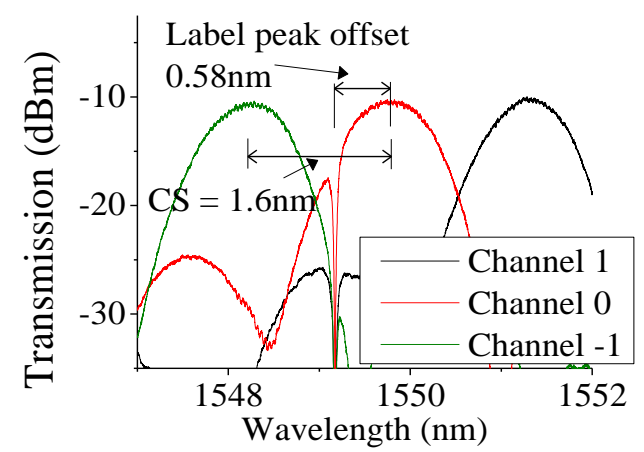

Fig. 3: Characterization of the cascaded demultiplexer and label extractor. Channel spacing

(CS) is equal to $1.6 \mathrm{~nm}$ or $200 \mathrm{GHz}$.

In Fig. 3, one can see a measured spectrum of the cascaded demultiplexer and label extractor which is used in this experiment. We will only evaluate the system with 3 payloads so only these channel responses are shown. The resonance peak of the MRR is clearly visible in the channel 0 response of the AWG. Due to waveguide dimension variations the exact position of the resonance is difficult to predict and active compensation will be necessary using integrated heaters to tune the resonance of the MRR ${ }^{6}$.

In this case the resonance peak of the label extractor has a peak offset of $0.58 \mathrm{~nm}$ from the center wavelength (channel 0 ) of the demultiplexer channel response.

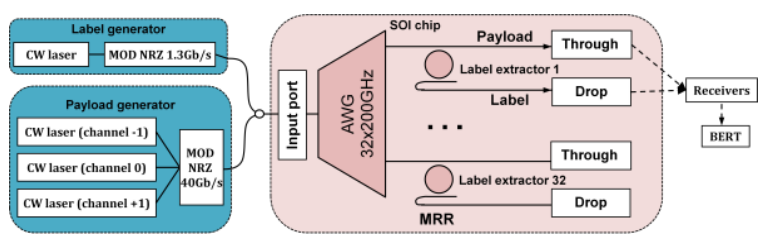

Fig. 4: Schematic of the experimental setup

Fig. 4 shows the experimental setup employed to demonstrate the packet demultiplexer and label extractor. The system operates as follows. Three carrier wavelengths $(1548.16 \mathrm{~nm}$, $1549.67 \mathrm{~nm}$ and $1551.15 \mathrm{~nm}$ ) are modulated at $40 \mathrm{~Gb} / \mathrm{s}$ and combined with one label modulated at $1.3 \mathrm{~Gb} / \mathrm{s}$ at $1549.08 \mathrm{~nm}$. The power of a single payload channel is $1.8 \mathrm{dBm}$ and the label is transmitted at power of $-6 \mathrm{dBm}$. The input spectrum of our experiment is plotted in Fig. 5.

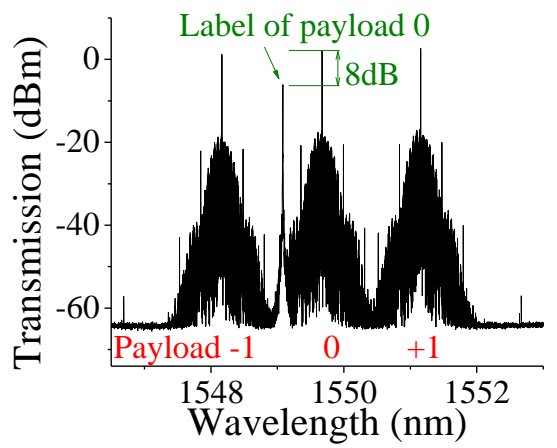

Fig. 5: Input of experiment. Three payloads at $40 \mathrm{~Gb} / \mathrm{s}$ with one in-band label to transfer the forwarding information to the switch.

The goal of our experiment is to successfully demultiplex the center payload channel (0) from the adjacent payload channels, and to extract error-free the in-band label of the middle payload without distorting the payload itself.

In Fig. 6, the spectrum of the through port of the label extractor (the extracted payload) is shown. One can see that both adjacent channels are successfully dropped with an ER of respectively $-20.4 \mathrm{~dB}$ and $-17.6 \mathrm{~dB}$. The label has an extra $23 \mathrm{~dB}$ drop with respect to the power of payload 0 , which is the combination of the ER of the ring, and the IL of the AWG (see Fig. 3). The IL of payload 0 is $14.8 \mathrm{~dB}$ which includes the IL of two fiber grating coupler (each $\pm 6 \mathrm{~dB}$ ), waveguide losses $(1 \mathrm{~dB})$ and $A W G(1.8 \mathrm{~dB})$. 


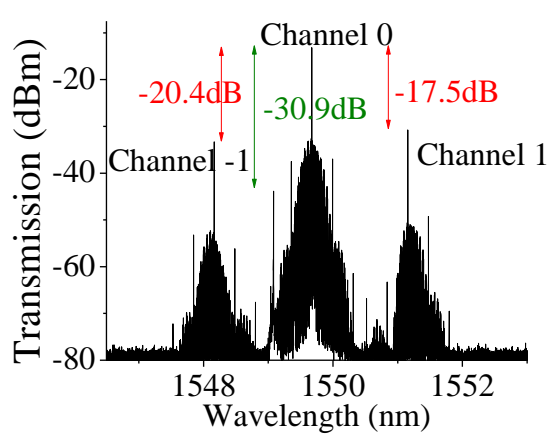

Fig. 6: Spectrum of the output through port of the label extractor.

In Fig. 7, we plot the spectrum at the drop port of the MRR, to check the spectrum of the label when transmitted through our component. One can find a $12 \mathrm{~dB}$ power ratio between the label and the payload of channel 0 , predicting a good signal to noise ratio of the label. The ratio between the label and the other payloads is more than $30 \mathrm{~dB}$.

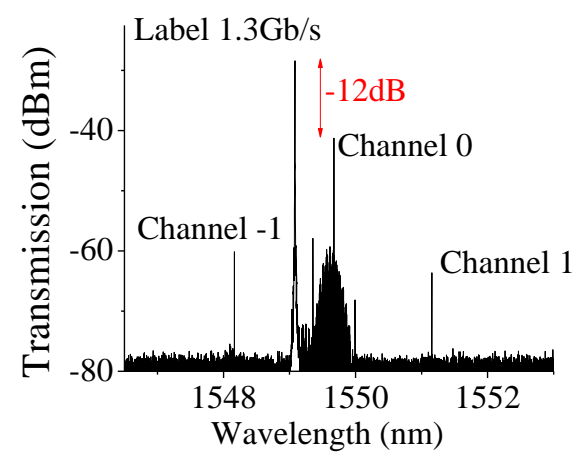

Fig. 7: Spectrum of the drop port of the label extractor.

This power ratio can be optimized with the peak offset of the microring resonance with respect to the carrier peak of the payload, for a given MRR and $A W G$ response and input power ratio between payload and label. The smaller the peak offset, the larger the crosstalk of the payload to the label but the larger the output power of the label. If the output power is not an issue, one can either decrease the input power of the label, or increase the peak offset between label and payload to further increase the quality of the label and the payload.

To evaluate the transmission quality of both payload and label, a BER curve is plotted in Fig. 8 and Fig. 9 respectively. One can notice for both signals error free operation with only a small power penalty of $\leq 0.5 \mathrm{~dB}$ compared with the back-to-back situation (not through the SOI chip).

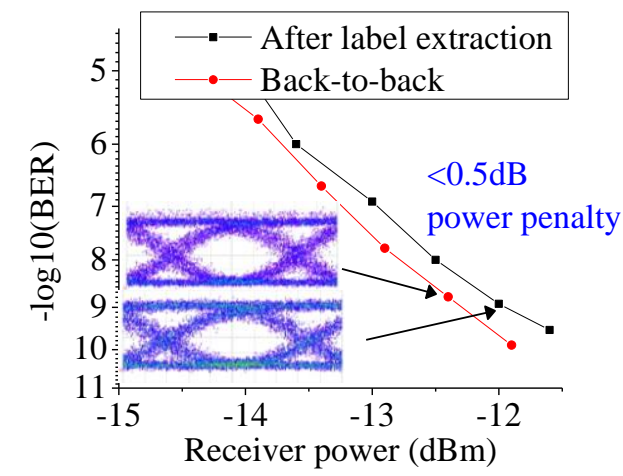

Fig. 8: BER curves of the $40 \mathrm{~Gb} / \mathrm{s}$ payload in back-toback and after the label extraction. In the inset eye diagram, you see the payload before the chip and after the label extraction.

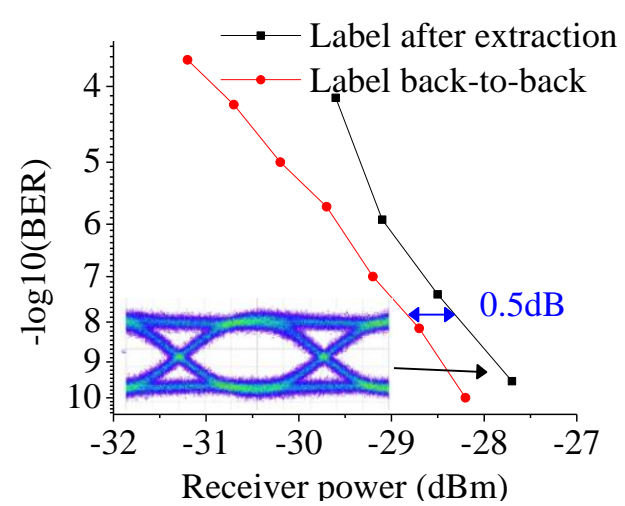

Fig. 9: BER curves of the label in back-to-back after the label extraction. In the inset eye diagram, you see the label after extraction.

\section{Conclusions}

We demonstrated a compact SOl-integrated $32 \times 200 \mathrm{GHz}$ AWG followed with a narrow-band MRR to establish the demultiplexing of three $40 \mathrm{~Gb} / \mathrm{s}$ payloads and the extraction of the inband optical label, both with power penalty of $\leq 0.5 \mathrm{~dB}$. This result is promising for the realization of a compact large port-count and low-latency photonic integrated optical packet switch.

\section{Acknowledgements}

The authors would like the ERC-project ULPPIC and FP7 LIGHTNESS project for supporting this work.

\section{References}

[1] C. Kachris, et.al., IEEE Com. Sur.\&Tut., 2012

[2] S. Di Lucente, et.al., Optical Express, 2012.

[3] J. Luo, et.al., Photonic Technol. Letters, 2012.

[4] P. De Heyn, et.al., Proc. ECIO, 2012

[5] S. Pathak, et.al., J. Lightwave Technol., 31(1), p.87-93 (2013)

[6] P. De Heyn, et.al., Proc. APC 2012, pp. 810, 2012. 